Jordan, C. 1969 , MNRAS, 142, 499

Jordan, C. 1970, MNRAS, 148, 17

Jordan, C. 1988a, J. Opt. Soc. Am. B, Vol. 5, No. 10, 2252

Jordan, C. 1988b, in Viotti, R., Vittone, A. \& Friedjung, M., eds, Physics of Formation of Fe II Lines outside LTE, p.223

Judge, P.G., Jordan, C. \& Feldman, U. 1992, ApJ, 384, 613

Kaastra, J.E. 1992, Internal SRON-Leiden Report, V2

Kurucz, R.L. 1988, Private Communication, results available on magnetic tape.

Landini, M. \& Monsignori Fossi, B.C. 1990, A\&AS, 82, 229

Lang, J. 1994, Special ed, Atomic Data Nucl. Data, Vol. 57

Mewe, R., Gronenschild, E.H.B.M. \& van den Oord, G.H.J. 1985, A\&AS, 62, 197

\title{
INTERPRETING THE SPECTRA OF CHEMICALLY PECULIAR STARS
}

\author{
THIERRY LANZ
}

\section{Universities Space Research Association NASA/GSFC, Greenbelt MD, USA}

Similarly to many astrophysical studies, a spectroscopic analysis of chemically peculiar (CP) stars requires extensive, accurate sets of atomic data. Our purpose is to show here that the CP stars can also serve as a laboratory for checking the accuracy of the atomic data. Despite additional challenges toward modeling the atmospheres of the CP stars, they have a definite advantage as laboratories for atomic physics: their strong chemical anomalies make some spectral features especially prominent in their spectra, which can be better checked against theoretical predictions. This statement may be applied to both to stars exhibiting large over- or under- abundances. We discuss several examples how CP stars can be used along this idea.

We will focus here on the chemically peculiar stars of the upper main-sequence, which encompass the classical Am and Ap stars. Chemical anomalies are widespread on the main sequence for spectral types B to F. These stars mostly show weak belium lines, and abnormally strong lines from iron-peak and heavier (e.g. the lanthanides) elements. A classical characteristics of Am stars in their weak $\mathrm{Ca}$ lines, while Ap stars may exhibit either strong $\mathrm{Si}$ or $\mathrm{Hg}$ lines. CP stars can be grouped according to the most obvious line strength anomalies, which are related to the effective temperature and to the presence or the absence of a magnetic field. In magnetic $C P$ stars, the chemical elements are not uniformly distributed over the stellar surface, but they seem to be concentrated (or depleted) in rings or polar caps following the magnetic geometry. Magnetic CP stars have also broad absorption features in their ultraviolet and visible spectra that can used photometric peculiarity criteria. Using the new extensive photoionization data from the Opacity Project (OP), the UV absorption features can be identified and the UV spectrum can be well 
reproduced.

The topics selected to illustrate the use of the atmospheres of CP stars as a checking laboratory are very accurate wavelengths and isotopic shifts, Zeeman pattern and line identification, and autoionization resonances. Superb quality, high-resolution spectra of the B9.5p HgMn star $\chi$ Lup obtained in the echelle mode with the Goddard High Resolution Spectrograph aboard HST have been presented by Leckrone et al. (1993, Phys. Scr. T47, 149). They showed that the internal wavelength accuracy of these spectra are of the order of 2-3 $\mathrm{m} \AA$. They have been able to firmly identify $\mathrm{Ru}$ II lines for the first time, but this required to improve the wavelengths of these lines to a similar accuracy. From the line positions and profiles, they also showed that the $\mathrm{Hg}$ II and the $\mathrm{Hg}$ III lines in the stellar spectrum are compatible only with a pure ${ }^{204} \mathrm{Hg}$ composition, though this is a minor isotope in the solar system mix. Mathys \& Lanz have suggested to use the Zeeman pattern in addition to the wavelength to secure line identification in the spectrum of magnetic stars showing Zeeman split lines. Conversely, the stellar spectra can be used to check Landé factors, which is especially interesting when intermediate coupling is important.

The strongest broad absorption feature, $\lambda 1400$, in the UV spectrum of ApSi stars has been attributed to silicon autoionization transitions already by Jamar et al. (1978, A\&A 63, 155). Artru et al. (1981, A\&A 96, 380) produced synthetic spectra with new atomic data for $\mathrm{Si}$ II, and they indeed predicted an absorption at $\lambda 1400 \AA$; however, they needed a very high silicon overabundance (100 times solar). Later on, Artru (1986, A\&A 168, L5) suggested that this stellar feature is in fact a single autoionization transition of $\mathrm{Si}$ II, $3 \mathrm{~s}^{2} 3 \mathrm{~d}^{2} \mathrm{D}-3 \mathrm{~s} 3 \mathrm{p}$ ( $\left.{ }^{1} \mathrm{P}\right) 3 \mathrm{~d}{ }^{2} \mathrm{~F}^{\circ}$, which was wrongly predicted at $\lambda 1221$ by previous atomic calculations. The OP calculations for Si II indeed predict this transition close to $\lambda 1400 \AA$. Using these photoionization cross-sections, we can now match the UV flux distribution of ApSi stars with a silicon abundance derived from the UV resonance lines. Besides the $\lambda 1400$ feature, there are several other spectral features which can be attributed to Si II thanks to the OP calculations: a broad absorption at $\lambda 1570 \AA$ is also due to a single autoionization transition; the flux below $\lambda 1310 \AA$ is almost completely absorbed due to an additional photoionization edge and several resonances. These new OP data are essential to interpret the UV spectrum of these stars. Conversely, this good theoretical match provides a direct, successful check of the reliability of the photoionization calculations in the OP database. Such a check has been previously possible only for a few ground states of neutral atoms for which laboratory data are available.

The OP database, TOPBASE, and of the Kurucz line list are of a tremendous importance for the analysis of stellar spectra. However, there are still few areas where progress can help to improve the spectroscopic work, especially for high-resolution studies. Starting from the OP data, we need (1) to include observed level energies to ensure exact wavelengths, (2) to have the fine structure, and (3) to improve the resolution for sharp autoionization resonances. For NLTE calculations, we need to know the contribution of each channel in the cross-sections given in TOPBASE, and similar quality data for collisional processes. Finally, these new data concern abundant light species; the data for heavier 
elements need to also to be much improved, since they are crucial for our understanding of various important nucleosynthetic processes in stars.

We have shown that the atmospheres of CP stars can be used to assess the new photoionization data provided by OP. Such an assessment is essential in view of the larger opacities obtained by $\mathrm{OP}$, which have allowed to solve several key problems in stellar astrophysics.

Nahar, S.N. \& Pradhan, A.K. 1994, J. Phys. B, 27, 429

Pradhan, A.K. \& Berrington, K.A. 1993, J. Phys. B, 26, 157

Raymond, J.C. 1988, in Pallavicini, R., ed, Hot Thin Plasmas in Astrophysics, Kluwer Ac.

Publ. Dordrecht, p.3

Smith, B.W., Raymond, J.C., Mann, J.B., Jr. \& Cowan, R.D. 1985, ApJ, 298, 898

\title{
THE OPACITY DRIVEN PULSATORS
}

\author{
WOJCIECH A. DZIEMBOWSKI \\ Copernicus Astronomical Center, ul. Bartycka 18, \\ 00-716 Warszawa, Poland
}

The opacity mechanism is the only well understood excitation mechanism of observed stellar oscillations. The driving effect arises due the a specific form of the opacity perturbation which leads to a conversion of the radiative flux energy into kinetic energy of oscillations. In 1963 Baker and Kippenhan demonstrated that the opacity mechanism acting in the HeII ionization zone is the cause of the Cepheid pulsations. Subsequently it has been shown that the same mechanism works in a large variety of stars. There are three opacity bumps that play a role in pulsation excitation in various types of objects - one connected with the $\mathrm{H}$ ionization, one connected with $\mathrm{He}$ II ionization zone - and one, discovered quite recently by Iglesias, Rogers \& Wilson (1988), caused by a large agglomeration of metal element (mostly $\mathrm{Fe}$ ) lines.

I focus on Main Sequence pulsators. There is a considerable difference among these stars in the manifestation of pulsation instability. However in all four types of Main Sequence pulsators - ro (rapidly oscillating) Ap, $\delta$ Scuti, $\beta$ Cephei, and SPB stars - a common feature is their multiperiodic variability. FG Vir a $\delta$ Scuti, having 10 modes definitely detected (Breger (1984)), is the best object in the class.

The observed variability in roAp stars is caused by excitation of high-order p-modes with frequencies similar to those of solar oscillation. The amplitudes, however, are by three orders of magnitude higher. The excitation is most likely due to the opacity bump in the H I 